\title{
Metabolome Analysis of Erythrocytes from Patients with Chronic Hepatitis C Reveals the Etiology of Ribavirin- Induced Hemolysis
}

\author{
Tetsuru Karasawa1 ${ }^{1}$, Takafumi Saito ${ }^{\bowtie}$, Yoshiyuki Ueno리 ${ }^{1}$ Masahiro Sugimoto², Tomoyoshi Soga² \\ 1. Department of Gastroenterology, Yamagata University School of Medicine, Yamagata, Yamagata 990-9585; \\ 2. Institute for Advanced Biosciences, Keio University, Tsuruoka, Yamagata 997-0052, Japan.
}

$\triangle$ Corresponding author: Takafumi Saito, M.D., Department of Gastroenterology, Yamagata University School of Medicine, 2-2-2 iida-nishi, Yamagata, Yamagata 990-9585, Yamagata, Japan Tel: +81-23-628-5309 Fax: +81-23-628-5311 Email: tasaitoh@med.id.yamagata-u.ac.jp

(c) Ivyspring International Publisher. This is an open-access article distributed under the terms of the Creative Commons License (http://creativecommons.org/ licenses/by-nc-nd/3.0/). Reproduction is permitted for personal, noncommercial use, provided that the article is in whole, unmodified, and properly cited.

Received: 2013.04.10; Accepted: 2013.07.22; Published: 2013.09.09

\begin{abstract}
Ribavirin is one of the major agents used in combination therapy with interferon for chronic hepatitis C, but is often associated with hemolytic anemia as a serious adverse event. Employing metabolome analysis, we demonstrated that the concentrations of intermediate metabolites produced by glycolysis and the pentose phosphate cycle in patients' erythrocytes were significantly decreased after administration of ribavirin. Our findings suggest that hemolysis associated with ribavirin is triggered by an energy crisis and consequent oxidative stress, thus having implications for the prevention of such hemolysis.
\end{abstract}

Key words: HCV, ribavirin, anemia, metabolome, glycolysis, pentose phosphate cycle.

\section{Introduction}

Hepatitis $\mathrm{C}$ virus (HCV) is the major causative pathogen of chronic liver diseases with a risk of progression to cirrhosis and hepatocellular carcinoma. Currently, ribavirin is one of the major agents used in combination therapy with interferon for chronic hepatitis C. During this treatment, however, patients sometimes suffer ribavirin-induced hemolytic anemia as an adverse event, necessitating discontinuation of the therapy or reduction of the dosage of anti-viral agents $(1,2)$.

The mechanism of the hemolysis induced by ribavirin has not been clearly determined, but oxidative stress and subsequent membrane fragility of erythrocytes have been suggested to play an important role $(3,4)$. After administration, ribavirin is converted to its phosphorylated form within the cell using adenosine triphosphate (ATP). However, phosphorylated ribavirin is not converted back to the dephosphorylated form because erythrocytes lack the enzymes necessary for dephosphorylation. Thus, the level of ATP in cells is reduced as a result of ribavirin phosphorylation $(4,5)$. The metabolic system in erythrocytes consists of glycolysis and the pentose phosphate cycle, for which ATP is essential. The pentose phosphate cycle begins with glucose-6-phosphate (G6P), which is the first intermediate metabolite produced by glycolysis, and thus it interacts with glycolysis closely. It has been hypothesized that deficiency of ATP causes impairment of the glycolytic system, thus triggering an energy crisis within erythrocytes, and inducing oxidative stress, leading to membrane fragility through impairment of the pentose phosphate cycle.

In the present study, to investigate changes in the intermediate metabolites of glycolysis and the pentose phosphate cycle in erythrocytes, we carried out the metabolomics analysis of erythrocytes from patients with chronic hepatitis $C$, who had received antiviral therapy with pegylated interferon plus ribavirin. It was anticipated that this approach would 
help to clarify the mechanism responsible for ribavirin-induced hemolysis.

\section{Methods}

Eight patients with chronic hepatitis C (4 men and 4 women, aged 54.1 $\pm 11.6 \mathrm{yr}$, mean \pm standard deviation) were treated with pegylated interferon plus ribavirin in accordance with the Japanese standard guideline for treatment of chronic hepatitis C (6). Erythrocytes were collected from the patients before and one week after the start of treatment, separated from whole blood by centrifugation, and resuspended in normal saline solution at a concentration of $400 \times$ $10^{6} / \mu 1$. For disruption of the erythrocyte membranes, $20 \mu \mathrm{M}$ methanol solution was added to $40 \mu \mathrm{l}$ of erythrocyte suspension. The samples were dissolved in $200 \mu \mathrm{M} 3$-aminopyrrolidine and trimesate. The levels of intermediate metabolites in erythrocytes were measured by capillary electrophoresis-time-of-flight mass spectrometry (CE-TOFMS) as reported previously (7-9). Informed consent was obtained from all patients, and the study was approved by the institutional ethics committee. Wilcoxon matched-pairs signed rank test was used for statistical analysis, and differences at $\mathrm{p}<0.05$ were considered to be significant.

\section{Results and Discussion}

Hemolytic anemia induced by ribavirin is one of the serious adverse events associated with therapy for chronic hepatitis $C(1,2)$. The erythrocyte count observed in this study showed a significant reduction at 2 weeks after the start of ribavirin administration $\left(452.0 \pm 43.9 \times 10^{6} / \mu 1\right.$ vs. $421.7 \pm 52.4 \times 10^{6} / \mu 1$ : pretreatment vs. 2 weeks after treatment, mean \pm standard deviation, $\mathrm{p}=0.028$ ). The drop in the erythrocyte count progressed and became $388.1 \pm 58.0 \times 10^{6} / \mu l$ at 4 weeks after the start of ribavirin administration. Although the mechanism of this hemolysis has not been precisely clarified, a reduction of ATP due to ribavirin phosphorylation has been suggested (10). A recent study showed that ribavirin-induced anemia is primarily due to the effect of the drug on guanosine triphosphate, consequently leading to a reduction of ATP in erythrocytes (11). To clarify this mechanism, we focused on the levels of intermediate metabolites of glycolysis and the pentose phosphate cycle in erythrocytes.

The changes in the levels of intermediate metabolites belonging to the glycolytic system and the pentose phosphate cycle in erythrocytes between samples collected before and after ribavirin administration are shown in Table 1. As ribavirin was taken up by erythrocytes, ATP was consumed for its phosphorylation, and as erythrocytes lack the enzymes necessary for ribavirin dephosphorylation, ATP deficiency progressed and eventually inhibited the glycolytic system. Here we found that the levels of the intermediate metabolites of glycolysis in erythrocytes, including G6P, fructose-6-phosphate, 2, 3-diphosphoglyceric acid and 3-phosphoglyceric acid, were significantly lower one week after ribavirin administration than before the start of treatment. This suggested that the energy metabolism of erythrocytes had been impaired by ribavirin.

Table I. Levels of intermediate metabolites for glycolytic system and pentose phosphate cycle in erythrocytes of patients receiving ribavirin (fmol/cell).

\begin{tabular}{lccc}
\hline & $\begin{array}{c}\text { Erythrocytes } \\
\text { before treatment }\end{array}$ & $\begin{array}{c}\text { Erythrocytes } \\
\text { one week of treatment }\end{array}$ & p-Value $^{\mathrm{a}}$ \\
\hline Glycolytic system & & & \\
Glucose-6-phosphate $^{\mathrm{b}}$ & $12.8 \pm 7.9 \times 10^{-4}$ & $8.2 \pm 4.7 \times 10^{-4}$ & $0.036^{*}$ \\
Fructose-6-phosphate $^{\mathrm{b}}$ & $6.1 \pm 4.8 \times 10^{-4}$ & $1.3 \pm 2.4 \times 10^{-4}$ & $0.028^{*}$ \\
Fructose-1,6-diphosphate & $1.9 \pm 0.7 \times 10^{-2}$ & $1.5 \pm 0.6 \times 10^{-2}$ & 0.069 \\
2,3-Diphosphoglyceric acid & $3.2 \pm 0.7 \times 10^{-1}$ & $2.6 \pm 0.9 \times 10^{-1}$ & $0.043^{*}$ \\
3-Phosphoglyceric acid & $3.0 \pm 0.5 \times 10^{-3}$ & $2.3 \pm 0.5 \times 10^{-3}$ & $0.017^{*}$ \\
Pentose phosphate cycle & & & \\
6-Phosphogluconic acid & $2.9 \pm 3.2 \times 10^{-4}$ & $1.2 \pm 2.2 \times 10^{-4}$ & 0.068 \\
D-Ribose-5-phosphate & $4.2 \pm 1.6 \times 10^{-3}$ & $3.1 \pm 0.9 \times 10^{-3}$ & $0.012^{*}$ \\
D-Sedoheptulose-7-phosphate & $3.3 \pm 2.5 \times 10^{-4}$ & $1.1 \pm 2.4 \times 10^{-4}$ & 0.091 \\
\hline
\end{tabular}

Wilcoxon matched-pairs signed rank test. ${ }^{\mathrm{b}}$ These are related to pentose phosphate cycle.

Data are expressed as mean \pm standard deviation. $*$ Significamt. 
G6P is the key metabolite that is central to both glycolysis and the pentose phosphate cycle in erythrocytes. G6P is metabolized to 6-phosphogluconic acid, D-ribose-5-phosphate and D-sedoheptulose-7phosphate to supply nicotinamide adenine dinucleotide phosphate (NADPH) for the pentose phosphate cycle. The levels of these metabolites in patients' erythrocytes were all reduced after ribavirin administration, among which that of D-ribose-5-phosphate became significantly lower. This indicated that the decrease in the level of G6P influenced the activity of the pentose phosphate cycle and caused a decrease of NADPH. NADPH has an important role in the reduction of glutathione (GSH) in concert with glutathione reductase. Thus it was suggested that the decrease of NADPH resulting from impairment of the pentose phosphate cycle led to a decrease in the concentration of reduced GSH, consequently increasing the degree of oxidative stress in erythrocytes. In fact, a previous study has shown that ribavirin treatment causes a significant increase of malondialdehyde and methemoglobin in erythrocytes, both being typical products of oxidative damage (4). Taken together, the data suggest that two major events in erythrocytes - an energy crisis due to ATP deficiency in the glycolytic system and consequent oxidative stress due to impairment of the pentose phosphate cycle - are closely related to the occurrence of hemolysis associated with ribavirin.

The single nucleotide polymorphisms (SNPs) of inosine triphosphatase (ITPA) gene are shown to be strongly associated with ribavirin-induced hemolytic anemia in chronic hepatitis C (12). Further studies are needed to investigate a relation between SNPs of ITPA gene and levels of intermediate metabolites for glycolytic system and pentose phosphate cycle in a larger cohort of patients. In the treatment of chronic hepatitis C, adequate doses of ribavirin are closely associated with viral clearance, and prevention of both viral breakthrough and emergence of resistant variants (2). For achieving the maximum effect of antiviral therapy, strategies for anemia management during ribavirin administration are essential. The results of this study suggest that some form of medication strategy for both the energy crisis and oxidative stress caused by ribavirin may be important for prevention of erythrocyte hemolysis.

\section{Abbreviations}

HCV: Hepatitis C virus; ATP: adenosine triphosphate; G6P: glucose-6-phosphate; CE-TOFMS: capillary electrophoresis-time-of-flight mass spectrometry; NADPH: nicotinamide adenine dinucleotide phosphate; GSH: glutathione; SNPs: single nu- cleotide polymorphisms; ITPA: inosine triphosphatase.

\section{Acknowledgement}

We thank Prof. Dr. Sumio Kawata for valuable advises on this work. This study was supported in part by grants from Japan Science and Technology Agency, and also in part by grants from the Ministry of Health, Labor and Welfare of Japan.

\section{Competing Interests}

The authors have declared that no competing interest exists.

\section{References}

1. Hynicka LM, Heil EL. Anemia management in patients with chronic viral hepatitis C. Ann Pharmacother. 2013; 47 :228-236.

2. Jacobson IM, Kowdley KV, Kwo PY. Anemia management in the era of triple combination therapy for chronic HCV. Gastroenterol Hepatol (N Y). 2012; 8 (9 Suppl 6) : 1-16.

3. Grattagliano I, Russmann S, Palmieri VO, et al. Glutathione peroxidase, thioredoxin, and membrane protein changes in erythrocytes predict ribavirin-induced anemia. Clin Pharmacol Ther 2005; 78: 422-432.

4. De Franceschi L, Fattovich G, Turrini F, et al. Hemolytic anemia induced by ribavirin therapy in patients with chronic hepatitis $C$ virus infection: Role of membrane oxidative damage. Hepatology 2000; 31: 997-1004.

5. Page T, Connor JD. The metabolism of ribavirin in erythrocytes and nucleated cells. Int J Biochem 1990; 22 : 379-383.

6. Editors of the Drafting Committee for Hepatitis Management Guidelines: The Japan Society of Hepatology. Guidelines for the Management of Hepatitis C Virus Infection. First edition, May 2012, The Japan Society of Hepatology. Hepatol Res 2013; 43: 1-34.

7. Soga T, Baran R, Suematsu M, et al. Differential metabolomics reveals ophthalmic acid as an oxidative stress biomarker indicating hepatic glutathione consumption. J Biol Chem 2006;281:16768-76.

8. Shintani T, Iwabuchi T, Soga T, et al. Cystathionine beta-synthase as a carbon monoxide-sensitive regulator of bile excretion. Hepatology 2009;49:141-50

9. Soga T, Sugimoto M, Honma M, et al. Serum metabolomics reveals $\gamma$-glutamyl dipeptides as biomarkers for discrimination among different forms of liver disease. J Hepatol 2011;55:896-905

10. Lau JY, Tam RC, Liang TJ, et al. Mechanism of action of ribavirin in the combination treatment of chronic HCV infection. Hepatology 2002; 35: 1002-1008.

11. Hitomi Y, Cirulli ET, Fellay J, et al. Inosine triphosphate protects against ribavirin-induced adenosine triphosphate loss by adenylosuccinate synthase function. Gastroenterology 2011; 140:1314-1321.

12. Fellay J, Thompson AJ, Ge D, et al. ITPA gene variants protect against anaemia in patients treated for chronic hepatitis C. Nature 2010; 464: 405-408. 